\section{Cardiac stress test}

\author{
KATHLEEN A. KUK
}

Heart disease continues to be the nation's number one health problem, leading all other illnesses in terms of economic burden, restricted activity, hospital confinement, and death. In light of these dire consequences, thousands of health-conscious Americans are queuing up for a screening test that may serve as an "early warning system" for heart disease.

The cardiac stress test (also known as a stress electrocardiography or graded exercise test) is painless. It requires no drugs, probes, or needles, and can be performed within 30 minutes in a doctor's office or an outpatient clinic of a hospital. Yet the test is an imperfect tool at best...and does carry a small but real risk of triggering a heart attack. This article reviews the basic aim, risks, and ideal candidates for cardiac stress testing.

\section{Transforming impulses into data}

A stress test puts the heart muscle through its paces. The great oxygen demands created by riding a bicycle or walking along a treadmill may reveal irregularities of the heart that might otherwise go unnoticed. The test owes its existence to the ability of the electrocardiogram (ECG) machine to capture fleeting electrical impulses and transform them into hard data.

Each normal heartbeat originates with an electrical "spark" from a specialized section of the heart. This impulse is transmitted outward and can be detected by electrodes placed on the skin's surface. A computer then analyzes the information and creates a jagged graph of distinctive peaks and valleys. Most heart disease, including heart attacks, irregular heart rhythms, and inflammation of the heart muscle, causes an abnormal ECG pattern. During a stress test, physicians hope to induce these quirks - in a controlled environment, of course - and identify those persons at risk of an impending heart attack.

\section{Four functions}

A stress test performs four basic functions. It can confirm a diagnosis of heart disease in patients who have suffered unexplained episodes of chest pain, shortness of breath, palpitations, or dizziness. A stress test can evaluate the progress of patients recovering from a previous heart attack or recent surgery. It can screen the population who displays no symptoms...yet are at risk of heart disease because of heredity or lifestyle. And, finally, a stress test can reassure healthy but sedentary individuals who are about to embark on a rigorous exercise program.

\section{How the test is performed}

Upon arriving in a T-shirt, shorts, and sneakers, patients will be attached to the ECG machine by cables attached to adhesive electrodes. The skin will be gently cleansed, shaved (if necessary), abraded, and coated with a gel so that the adhesive bound electrodes will have good contact to adhere. A number of electrodes will be placed at preselected sites on the chest. This is performed so that the ECG machine can "look" at the heart from several different directions or "leads." There is no danger that the electrodes will transmit a shock; in fact, the patient will not even be able to detect the current.

Before the test begins, the physician (or technician) will obtain baseline readings of the ECG, pulse rate, and blood pressure. These "before" readings will be compared with the "during" and "after" results. 
The patient will then carefully mount the slowly moving treadmill track. He should carefully grasp the support railings to maintain balance, not to support weight. If the physician opts for the bicycle test, the seat and handle bars should be adjusted beforehand. Once again, the handle bars should be gripped lightly for balance.

Both tests begin slowly. But what started out as a stroll turns into a jog as the treadmill speeds up and gains an upward slant. The biker, too, finds himself pumping harder to meet greater pedestal resistance.

During this time, the ECG, heart rate, and blood pressure are being constantly monitored by a physician. He will stop the test immediately if abnormal ECG patterns develop or if blood pressure suddenly plummets or sky-rockets. The ultimate end-point of this trial is to reach one's predetermined "target heart rate." Often, however, the test is self-limited, and the patient is forced to stop because of exhaustion, shortness of breath, or chest pain. The complete test lasts roughly a half hour.

The pace of exercise slows so the patient has time to "cool down." The patient is then helped to a chair, where blood pressure and heart rate are monitored for an additional 10 to 15 minutes.

Printouts of ECG readings are available almost immediately. However, the physician will need time to scan the graphs and analyze their interpretations. A follow-up visit will probably be scheduled to discuss the findings and plan therapeutic intervention, if needed. If the results are inconclusive, a repeat stress test or a more sophisticated procedure may be called for.

\section{Risks}

Obviously, there are some inherent risks involved in subjecting the heart to so much stress. For- tunately, the danger is minimized with careful monitoring and constant physician supervision. Studies show that the odds of suffering a heart attack while undergoing a stress test are less than 1 in 500; the chance of dying from the attack is less than 1 in 10,000 .

Meticulous pre-selection of patients has also reduced the gamble. The procedure is off limits to patients with unstable angina (chest pain), unpredictably irregular heartbeats, inflammation of the heart muscle, uncontrolled high blood pressure, or several other cardiac disorders. In addition, the test cannot be performed by persons who have certain lung problems or physical handicaps that restrict movement.

\section{Candidates}

The decision to go ahead with a stress test may be very clear cut for a patient with suspicious symptoms or confirmed diagnosis of heart disease. But what of the weekend athlete who is a tad out of shape but, on the whole, feeling fine? At one time, blanket approval of the test was granted to virtually anyone over the age of 35 . Today, the American Heart Association and The American College of Cardiology recommend that physicians review each individual case.

Generally, a stress test should be strongly considered for any person who has two or more of the following risk factors:

- Age over 40 years

- Male sex

- Strong family history of heart disease, in which a close relative suffered or died of a heart attack before the age of 55

- Serum cholesterol level $>220 \mathrm{mg} . / \mathrm{dl}$.

- Blood pressure $\leqslant 160 / 90 \mathrm{~mm}$. hg

- Cigarette smoking

- Diabetes mellitus 
It should be noted, however, that roughly 40 percent of the population is "at risk" on the basis of age and sex alone. Another 40 percent have only one additional risk factor. Therefore, the test is an absolute must for only a minority of the country.

The test may be considered for any man over age 40 or woman over age 50 , regardless of risk factor, who plans more spirited romps than a simple round of doubles tennis or golf. A look at current pursuits can also provide clues. If he or she regularly performs such low-level activities as climbing several flights of stairs or mowing the lawn without chest tightness or fatigue, then chances are the stress test may be unnecessary. A better recommendation might be to embark on a gradual exercise program that highlights walking and frequent pulse-taking.

The cardiac stress test is usually not recommended for fit adults in their early 20 s and 30 s. A periodic check-up to evaluate cardiac risk factors is more appropriate.

\section{A healthy alternative}

Controversy still reigns as to whether or not an apparently healthy individual should receive the cardiac stress test "just to be safe." The consensus seems to hold that annual stress testing after age 50 to 55 is "prudent." Still, the test is not without flaws. Certain medications may mask or alter test results. Worse still, the test may fail to reveal symptomless, "silent" heart disease-a possible forerunner of sudden cardiac death.

A practical and healthy alternative is to act today to reduce cardiac risk factors of tomorrow. Stop smoking, lose weight, control high blood pressure, reduce cholesterol levels, and learn how to manage stress!

(All material in the Patient Health Guide is reviewed by JAOA physician referees.)

\section{References}

Complete Book of Medical Tests. By M.A. Moskowitz and M.E. Osband. W.W. Norton \& Company, New York, 1984

Diagnostic Nursing84 Books. Springhouse Corporation, Springhouse, PA, 1984

Graded exercise testing. By S.S. Gubin and R.D. Judge. American Family Physician, vol. 35, pp. 123-9, Apr 1987

Ischemic heart disease, by E. Braunwald and P.F. Cohn. In Harrison's Principles of Internal Medicine. Edited by R.G. Petersdorf, et al. Ed. 10, McGraw-Hill, New York, 1983

Outpatient management of coronary artery disease. By J.F. Bingle and H.E. Mayhew. American Family Physician, vol. 36, pp. 191-200, Jul 87

Which adults need preexercise stress tests? By R.D. Conn and H.S. Miller, Jr. Patient Care, vol. 21, pp. 50-9, 28 Feb 1987 
